Chapter 17

\title{
Evolvement of microRNAs as \\ Therapeutic Targets for Malignant Gliomas
}

\author{
Sihan Wu, Wenbo Zhu and Guangmei Yan \\ Additional information is available at the end of the chapter \\ http://dx.doi.org/10.5772/52672
}

\section{Introduction}

Malignant glioma (MG), consisting of anaplastic glioma (AA, WHO grade III) and glioblastomamultiforme (GBM, WHO grade IV, 60-70\%), is the most common and destructive brain tumor [1]. Despite systemic treatment, surgical resection followed by radiotherapy and chemotherapy, the prognosis of patients with GBM remains poor with a median survival of 12 to 15 months and a 5-year survival rate of $9.8 \%[2,3]$. The dismal outcome fuels the need to understand the molecular basis of gliomagenesis and identify novel therapeutic targets for MG treatment.

With a developing understanding of core aberrant signaling pathways in MG, molecular targeted therapy has shown certain promise as a more rational strategy. Established targets, including receptor tyrosine kinases (RTK) such as EGFR, PDGFR and VEGFR, as well as mTOR, farnesyl transferase and PI3K, have drawn particular interests both in fundamental and clinical research. However, single targeted agents so far have not displayed desirable clinical outcomes as expected, with response rates of 0 to $15 \%$ and no prolongation of 6 month progression-free survival [4-6]. This disappointing outcome is primarily due to the nature of cancer with multi-genetic abnormality and inter/intra-tumor heterogeneity. Therefore, multiple targeting strategies are essential to achieve better clinical benefits.

MicroRNA (miRNA), a type of endogenous small non-coding RNA which negatively modulates gene expression in post-transcriptional level, has rapidly become a topical issue since it was firstly discovered in C. elegans two decades ago[7]. Increasing researches have reported that dysregulation of miRNA is closely associated with tumorigenesis and tumor progression. In gliomas, a group of miRNAs have been characterized as oncogenic and tumor-suppressive molecules. With a unique feature of multi-targeting, miRNA may become a new powerful weapon fighting against MG. Although much remains to be learned about formu- 
lating miRNAs as nucleic acid drugs, we feel that identification of miRNAs as therapeutic targets for gliomas is now within sight.

In this chapter, we will critically review issues relevant to contemporary researches on miRNAs and MG. Based upon other teams' and our data, this review will present findings on oncogenic and tumor-suppressive properties of miRNA in MG, focusing on the mechanisms of dysregulation, the involvement with core pathways of MG and the functional heterogeneity in different context. Besides, we will formulate evidences for miRNA-based clinical application in diagnosis and therapeutics of MG. The developing cognition of miRNAs will ultimately lead to innovative clinical approaches for MG.

\section{Oncogenic and tumor-suppressive miRNAs in malignant gliomas}

The very first oncogenic miRNA indentified in GBM is miR-21 as early as 2005 [8], although whose target genes were not fully understood at that time. In 2008, another group identified a set of targets of this miRNA which constitute a tumor-suppressive network including TGF- $\beta$, p53 and mitochondrial-apoptotic pathways [9]. This is one of the examples showing the multi-targeting nature of miRNAs in oncology. Beyond multi-targeting, these findings also prompt a hypothesis that miRNAs may have pathway preference in some cases.

Coincidently, also in 2005, S.A. Ciafreet al. firstly described that brain-enriched miR-128 and miR-181 family were frequently down-regulated in primary GBMs and cell lines [10], whose tumor-suppressive roles of these miRNAs were subsequently validated in the next few years [11-14].

In 2008, a milestone has been made in glioma research, accomplished by The Cancer Genome Atlas (TCGA) Research Network. Through integrative analysis of DNA copy number, gene expression and DNA methylation aberrations, TCGA defined three major pathways in GBM, which are RTK, p53 and RB signaling pathways, as shown in Figure 1 [15]. These pathways cannot be more familiar to every oncologist: frequently activated RTK signaling in cancer supports cell proliferation and survival, whereas p53 and RB signaling monitor cell cycle transition and couple to apoptosis and senescence pathways, which are commonly suppressed in cancer. To date, increasing oncogenic and tumor-suppressive miRNAs in MGs have been uncovered. In combination with genomic sketch of GBM described by TCGA, we have a deeper insight into the interactions within the core pathways of MG, which are controlled in coding and non-coding levels (Figure 1).

Here we do not pay our attention to the detail of each single miRNA, since several reviews have provided considerable details about their expression and function [16-18]. In this paper, we prefer to focus on how these miRNAs become aberrant, and which malignant phenotypes they contribute to.

As well as every protein coding gene, non-coding miRNA genes may similarly undergo over or lost expression, amplification, deletion, insertion, translocation and mutation, which lead to aberration of miRNAs. The mechanisms of miRNA aberration have not yet been ex- 
tensively studied. Nevertheless, there have been some clues showing that transcriptional activity, single nucleotide polymorphism (SNP) and chromosome deletion contribute to aberrant genotype and malignant phenotype of MG.

The most interesting research addressing miRNA transcriptional regulation machinery in MGs is the TP53 and miR-25/32 feedback circuitry [19]. Carlo M. Croce's and co-workers found that miR-25 and miR-32 are negatively regulated by TP53 via repressing their transcriptional factors $M Y C$ and E2F1. Intriguingly, by directly targeting MDM2 and TSC1, these miRNAs help stabilize P53 protein and suppress tumorigenicity in U87 GBM cells. Thus, a fine tuned recurrent autoregulatory circuit form. However, in the non-functional mutant TP53 context, miR-25/32 support cell proliferation per contra. These findings not only provide insights into the interaction between miRNAs and TP53 tumor-suppressive pathway, but also hint that a given miRNA may have distinct function in different genetic and biochemical status.

It is unexpected that miRNA genes rarely exhibit SNP or mutation within the major mature sequence segment, although which can be commonly found in the precursor (including the minor mature sequence which is formerly called the star sequence) and primary miRNA segments. Through mining human SNP database, Peng Jin's group identified a G>U polymorphism located in the eighth nucleotide of the mature miR-125a-5p. The U allele blocks the processing from pri-miRNA to miRNA precursor by altering the secondary structure, and impairs miRNA-mediated translational suppression [20]. MiR-125s have been demonstrated to be tumor-suppressive in GBM [21-23]. However, such nucleotide alteration has not yet been described in glioma clinical data. The rs11614913 SNP, which locates in the segment producing minor mature miR-196a-3p (termed miR-196a* previously), was firstly found to be associated with the risk of glioma in Chinese population [24]. Recent clinical study indicates that the rs2910164 in segment containing minor mature miR-146a-3p sequence is also relative to the risk and prognosis in adult glioma [25]. These findings improve our understandings of miRNA-related pathogenesis and diagnostics in gliomas. Further research is required to fully comprehend the exact mechanisms underlying the relationship between miRNA gene SNPs and clinical outcomes, which may have huge clinical potential.

Chromosome alteration undoubtedly contributes to miRNA aberration. Particularly in diffuse astrocytoma (DA, WHO II) and AA, chromosome 7q32 is a hotspot that frequently amplifies. This region embraces eight miRNAs: miR-593, miR-129-1,miR-335, miR-182, miR-96, miR-183, miR-29a and miR-29b-1. Among these miRNAs, our group recently reported that miR-335 is the most striking target in AA [26]. We have demonstrated that miR-335 confers invasive and proliferative malignant phenotype on astrocytoma via targeting DAAM1. Disrupting miR-335 by cholesterylated miRNA inhibitor abrogates invasion and elicits apoptosis both in vitro and in vivo. Chromosome amplification in cancer often creates abnormal high level of coding and non-coding products that subsequently infests the well-balanced signaling. Currently, drug screening and discovery in oncology specifically focus on overexpressed protein and over-activated kinase. We believe that miRNA, such as miR-335, may also be the target for drug development in the future. 


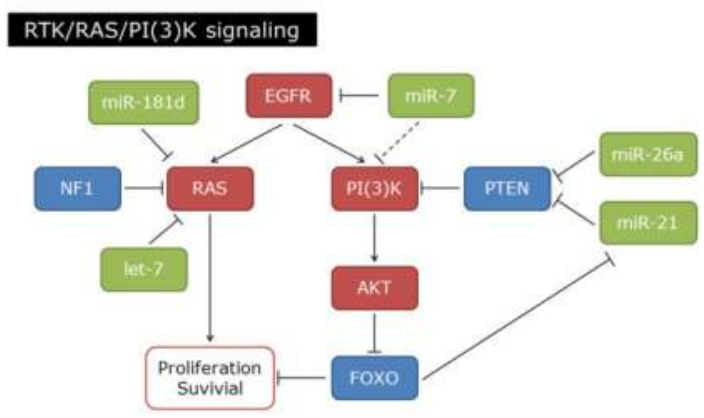

RB signaling

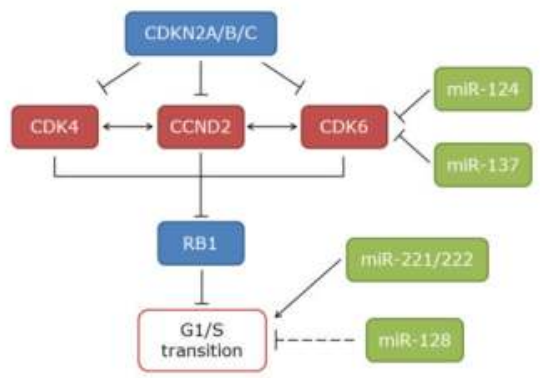

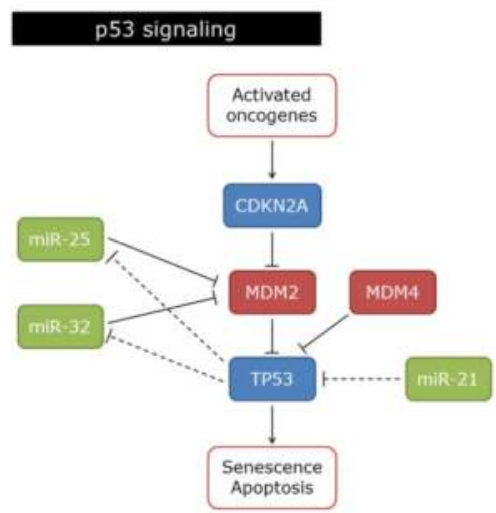

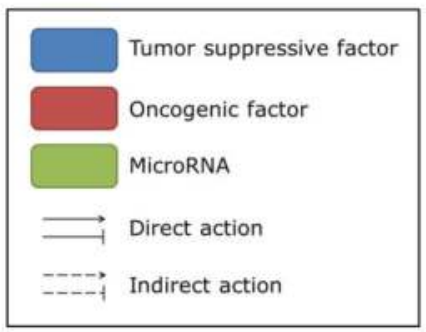

Figure 1. Interaction between microRNAs and core pathways in malignant gliomas.

\section{Dual scripts for a miRNA}

In the last session we have mentioned that miR-25/32 functions differently depended on TP53 status in GBM. It is not the sole evidence that a given miRNA plays distinct roles in respective models. In fact, miRNAs show complicated functional heterogeneity.

One of the examples is miR-135a/b. Remco Nagel et al. found increased expression levels of miR-135a and miR-135b during colorectal cancer progression, which target the tumor suppressor gene Adenomatous Polyposis Coli (APC)[27]. The oncogenic role of miR-135a was further confirmed by BR Zetter's group. They demonstrated that paclitaxel resistance is associated with up-regulation of miR-135a in multiple solid tumor cell lines. Knocking-down of miR-135a acquires susceptibility to paclitaxel[28]. However, in classic Hodgkin lymphoma (cHL), miR-135a directly regulates JAK2, thus suppressing the expression of BCL2L1 (also known as $B C L-X L$ ) and inducing apoptosis. Furthermore, higher level of miR-135a indicates better disease-free survival in cHL patients [29]. Likewise our group has also characterized that miR-135a functions as a selective killer of MG. We have reported that glial cell-enriched miR-135a is frequently down-regulated in MG and negatively correlates with 
the pathological grading of gliomas. Ectopic delivery of miR-135a selectively triggers mitochondrial dependent apoptosis in MG in vitro and in vivo. Interestingly, this lethal effect is neutral in normal glial cells and neurons [30].

MiR-335 gives us another example of the dual role nature of miRNAs. An early study identified that miR-335 suppresses breast cancer metastasis and migration through targeting SOX4 and TNC [31]. However, as mentioned above, our recent research found that this miRNA promotes invasion and proliferation in AA [26]. In this way, miRNAs seems to have cell-type-dependent functional heterogeneity. The definition of "tumor-suppressive" and "oncogenic" should be carefully employed depended on the cell types and diseases.

Even within the same cell type, the functional behavior of a given miRNA can also be diverse. Previously we have reported that activation of CAMP/PKA pathway, manipulated by Cholera toxin, induces cellular differentiation of MG, showing malignant phenotype reversion and potent GFAP (glial fibrillary acidic protein) expression [32]. As it has been well characterized, we found that IL-6/JAK2/STAT3 contributes to GFAP expression in this model [33]. Notably, epigenetic mechanism is also involved. In this context, the so-called oncogenic miR-335 in MG induced by Cholera toxin enhances GFAP expression in MG cell lines and primary cultures [34]. Multi-targeting is one of the natures of miRNAs. Signaling networks composed of target genes and their relative molecules may both contribute to tumorsuppressive and oncogenic function. It seems that the oncogenic functions of miR-335 are hindered under a high endogenous level of cAMP, rewiring to pro-differentiation pathways.

To sum up, the role of a given miRNA depends on the script which is assigned by the cell type, the genomic context and in part the cellular biochemical status. These observations remind that disease state as well as the genomic subclass of MGs should be considered in the preclinical study especially for drug discovery involved miRNAs.

\section{Involvement of miRNAs as novel biomarkers in malignant gliomas}

Biomarkers are distinctive biological indicators that serve for diagnosis, prognosis and medication. Traditional biomarkers include mRNAs, proteins and metabolites. At present, miRNAs are emerging as novel and practical biomarkers in oncology. Due to their surprising stability, miRNAs can not only be detected in fresh and frozen tissues, but also in body fluids and even formalin-fixed paraffin-embedded samples. In the study of Roland Schroers, miR-15b and miR-21 are found in cerebrospinal fluid and demonstrated to be markers of glioma [35]. Although much remains to be studied, e.g. to discover more accurate and specific markers for respective glioma types, this finding provides a new opportunity to develop less invasive and early diagnostic approach for gliomas.

Besides diagnosis, miRNAs may be promising biomarkers for prognosis. Jun Li's group has reported that miR-182 is markedly up-regulated in various glioma cell lines and primary glioma specimens. Furthermore, higher level of miR-182 is associated with poor overall survival of patients with malignant glioma [36]. A more comprehensive study was recently car- 
ried out by Sujaya Srinivasan, et al. They analyzed miRNA expression profile of GBM patients $(n=222)$ from TCGA database and found a ten-miRNA expression signature that predicts survival for GBM. In detail, three miRNAs (miR-20a, miR-106a and miR-17-5p) were identified as protective markers and seven miRNAs (hsa-miR-31, hsa-miR-222, hsamiR-148a, hsa-miR-221, hsa-miR-146b, hsa-miR-200b and hsa-miR-193a) were risky [37].

Recent studies have raised a possibility that miRNAs may function as predictive markers for therapeutics. Temozolomide (TMZ) is a well-known alkylating agent against gliomas, which methylates guanines in the O-6 position. Accumulating O-6 methyl guanines lead to DNA mismatch during replication and ultimately result in cell death. Although MGMT (methylguanine methyl transferase) promoter methylation has been proved to be a strongest marker to predict the TMZ response in low-grade and high-grade gliomas [38-40], it may not be the only indicator. Recent research indicates that miR-181b/c, miR-195, miR-455-3p and miR-10a-3p, rather than MGMT promoter methylation, predict response to concomitant chemoradiotherapy and acquired TMZ resistance in GBM cell line and patients [41, 42]. In addition, increasing evidences indicate that miRNAs also contribute to other drug response. For instance, up-regulated miR-21 confers teniposide and paclitaxel resistance on GBM cells [43, 44]. Various highly efficient MGMT inhibitors have been developed for sensitizing tumor to TMZ [45]. The emerging roles of miRNAs in drug resistance of TMZ and other antineoplastic agents give us a novel angle to include miRNAs as therapeutic targets.

\section{Perspectives on miRNAs as nucleic acid drugs in malignant gliomas}

\subsection{MiRNAs replacement therapy and suppressive therapy}

Human body has an extremely complicated internal environment, relying on balance and stability of which human keep their health. Once the homeostasis is disrupted by down-regulation or up-regulation of some important molecules including miRNAs, human will suffer from all kinds of diseases. Based on the disequilibrium status, the ideal protocols to cure diseases are believed to replace what is lost and to repress what is excessive, which are respectively called replacement therapy and suppressive therapy. For the former, estrogen replacement therapy is the most representative example, which is generally used in menopausal women with menopausal syndrome caused by ovarian hormones loss and has shown a positive effect [46]. For the latter, a typical case is a famous anti-angiogenesis drug Avastin, the humanized anti-vascular endothelial growth factor (VEGF) monoclonal antibody for cancer therapy [47]. Besides, Gefitinib and Glivec specially inhibiting EGFR tyrosine kinase, have both achieved high clinical benefit rates in treating cancer especially lung carcinoma and chronic myelocytic leukemia[48,49], further indicating the efficacy of suppressive therapy via targeting core molecules in tumorigenesis.

Notably, increasing evidences demonstrated that gliomagenesis is also closely associated with deregulation of miRNAs, such as up-regulation of oncogenic miRNAs and down-regulation of tumor suppressive miRNAs. Therefore, targeting these aberrant miRNAs has tremendous potential as a therapeutic strategy by down- or up-regulating altered genes. 
Specially, miRNA mimics can be synthesized to replace lessened ones critical in gliomagenesis, and miRNA inhibitors can be designed to suppress the function of up-regulated oncogenic miRNAs. Just less than two decades after discovering the role of miRNAs in oncology, the replacement and suppressive therapies of miRNA are already being used in preclinical researches. We have proved that artificially rectification of abnormally expressed miRNAs inhibited growth of glioma xenografts in mice and prolonged the median survival of rat with orthotopic glioma[26,30], opening a new sight for glioma treatment.

\subsection{MiRNA-targeting cocktail therapy}

Glioma is a complicated disease of altered genes. TCGA has reported multiple chromosomal aberrations, nucleotide substitutions and epigenetic modifications that construct three core pathways (as shown in Figure 1] [15]. Furthermore, glioma is a disease of altered miRNAs. A genome-wide expression profiling of 26 GBMs, 13 AAs and 7 normal brain samples identifies multiple deregulated miRNAs in MGs [50]. In addition, large scale of sequencing analysis of GBMs has identified extensive genetic heterogeneity between GBM samples from different patients [51], implying the possibility of miRNA's heterogeneity. The multiplicity of deregulated miRNAs and the heterogeneity of deregulated miRNAs expression profiling may foster therapeutic failure and tumor adaptation to solo miRNA-targeting therapy.

To date, researchers just verified the efficiency of targeting solo miRNA as if by prior agreement. What will happen if target several pivotal miRNAs simultaneously? Given the multiplicity of deregulated miRNAs in glioma, a cocktail consisting of special miRNA mimics and/or inhibitors may kill multiple birds with one stone. In another word, components in cocktail may have an additive effect or even a synergistic effect against MGs. Furthermore, considering individual differences, one cocktail may not fit for each patient with MGs. If we can design a specific miRNA cocktail based on individual deregulated miRNAs, miRNA individualized treatment will be within our sights. Certainly, additional preliminary evidences are needed to verify the feasibility and availability of miRNA-targeting cocktail therapeutics.

\subsection{Engineering miRNA}

Engineering miRNA (emiRNA) is artificially designed in sequence, endowed with functional similarity to natural miRNA and functional specificity according to human's will. In a sense, emiRNA is a branch of small interfering RNA (siRNA) that possesses multi-targeting power just as natural miRNA.

Leastwise, there are two strategies to design emiRNA: (a) adjustment based on the natural miRNA sequence; (b) de novo design.

In the sessions above, we have discussed the fact that a given miRNA may play distinct role in different models, e.g. different genetic background, different cell type and different second messenger level. Even in a "conclusive" circumstance that a certain miRNA strongly kills a type of cancer cell, we still need to evaluate its pharmacodynamics in the cancer tissue and toxic side effect to the normal tissue before clinical application. If a natural sequence 
miRNA shows remarkable antitumor effect but exhibits unsatisfying selectivity, does it mean we should totally abandon this target? The problem may be solved by emiRNA technology. Different dominant target genes and their roles in different models determine the functional heterogeneity of a given miRNA. If we are able to find out the undesired targets that lead to unsatisfying selectivity, perhaps by keeping the seed region of the miRNA and adjusting its $3^{\prime}$ nucleotide sequence, we could either avoid the interaction between the miRNA and the undesired target genes, or enhance the binding to our goal targets.

In broader context, we could design an artificial miRNA in a de nove way. Cancers are known as a type of multigenic disease. By comprehensive sequencing, RTK/RAS/PI[3]K, p53 and RBsignalings are characterized as the core pathways in GBM [15]. In virtue of bioinformatics, it is possible to design a miRNA that contemporaneously targets the key components of two or more pathways by carefully examining their mutual microRNA recognition elements (MREs).

However, numbers of scientific problems need to be addressed before putting emiRNA into practice. We still have limited knowledge about the details of miRNA biogenesis and the MRE language passing on along evolution. Someday the secret will be unlocked and emiRNA may be a practical weapon against cancers.

\subsection{MiRNA formulation}

Another attractive issue of translating miRNA into clinical application is formulation. Although whose length is quite short in the RNA world, miRNA as well as siRNA is a type of high molecular weight drug in pharmaceutical point of view. Additionally, electric charge contributed by phosphate groups of the nucleic acid backbone make it inadequate for CNS delivery. Fortunately, new technical progress brings miRNA formulation within sight.

In the earlier period, viral vector including retrovirus, adenovirus, herpes simplex virus and adeno-associated virus were broadly used due to their high efficiency for both gene delivery and expression. However, limitations associated with safety, genomic stability and immunogenicity motivate scientists to focus on non-viral systems. At present, remarkable achievements have been made in chemical modifications and conjugations of small RNA. For example, locked nucleic acid (LNA) technology not only increases melting temperature of RNA-RNA duplex thus enhancing the binding affinity between small RNA and target transcript, but also improves the half-life of small RNA in serum, which is critical for therapeutic use [52]. Cholesterylation, viz. modification with cholesteryl functionality, which improves the cellular uptake of small RNA, is now extensively used for miRNA mimics/inhibitors and siRNA in preclinical research [53]. Two reviews have provided clear and comprehensive introduction of the progress on chemical small RNA encapsulation [54, 55]. Particularly, a recent study from Paula Hammond and colleagues reported a self-assembled microsponges of small RNA hairpin polymersin corporated more than half a million copies of siRNA in a single nanoparticle [56]. This novel technology allow one thousand times lower concentration to achieve same degree of gene silencing efficiency as conventional strategies, showing great clinical application potential. In the near future, small RNA formulation will further benefit from evolution of nanotechnology and biomaterial technology. 


\section{Concluding remark}

Glioma is not the type of high incidence disease; however the depressive clinical outcomes still threaten human health. Special location, rapid proliferation, invasive growth and massive angiogenesis are the key issues that limit treatment for MG. In addition, extremely complicated histopathological and genomic classifications perplex fundamental and clinical research.

With the evolution of new generation high through-put sequencing technology, clearer insights of driver mutations and core pathways in various cancers are gradually revealed in the recent period [57-59]. According to these cancer genomic studies, we realize that not only aberrant coding genes lead to cancers, but also non-coding components underneath the iceberg. Twenty-year's research on miRNA opens a new world of genetics and oncology. More and more groups are dedicated to study the role of miRNA in cancer including glioma. Albeit fragmentary, accumulating data will offer deeper insights of glioma with more systematic considerations.

Compared with other non-coding protagonists, e.g. histone modification and DNA methylation, miRNA is the easiest role to be specifically manipulated, either by over-expression or inhibition, which determines miRNA to be the star with enormous clinical potential. Although we have not drawn an elaborate picture of miRNA biogenesis, especially how primary sequence and intra-cellular microenvironment influence secondary structure and eventually contribute to processing and maturation, as well as the communication principles between miRNA and MRE, we believe that technologies nowadays are poised to uncover the law of miRNA in oncology. We envision that this intrinsic biomolecule will be the therapeutic target for cancer.

\section{Author details}

Sihan Wu, Wenbo Zhu and Guangmei Yan

Zhongshan School of Medicine, Sun Yat-sen University, People's Republic of China

\section{References}

[1] Louis, D.N., Ohgaki, H., Wiestler, O.D., Cavenee WK. WHO Classification of Tumours of the Central Nervous System. IARC Press; 2007.

[2] Meyer M a. Malignant Gliomas in Adults. New England Journal of Medicine. 2008 Oct 23;359(17):1850-1850. 
[3] Wirth T, Samaranayake H, Pikkarainen J, Määttä A-M, Ylä-Herttuala S. Clinical trials for glioblastomamultiforme using adenoviral vectors. Current opinion in molecular therapeutics. 2009 Oct;11(5):485-92.

[4] Sathornsumetee S, Rich JN, Reardon DA. Diagnosis and treatment of high-grade astrocytoma. Neurologic clinics. 2007 Nov;25(4):1111-39, x.

[5] Chi AS, Wen PY. Inhibiting kinases in malignant gliomas. Expert opinion on therapeutic targets. 2007 Apr;11(4):473-96.

[6] Sathornsumetee S, Reardon DA, Desjardins A, Quinn JA, Vredenburgh JJ, Rich JN. Molecularly targeted therapy for malignant glioma. Cancer. 2007 Jul 1;110(1):13-24.

[7] Lee RC, Feinbaum RL, Ambros V. The C. elegansheterochronic gene lin-4 encodes small RNAs with antisense complementarity to lin-14. Cell. 1993 Dec;75(5):843-54.

[8] Chan J a, Krichevsky AM, Kosik KS. MicroRNA-21 is an antiapoptotic factor in human glioblastoma cells. Cancer research. 2005 Jul 15;65(14):6029-33.

[9] Papagiannakopoulos T, Shapiro A, Kosik KS. MicroRNA-21 targets a network of key tumor-suppressive pathways in glioblastoma cells. Cancer research. 2008 Oct 1;68(19):8164-72.

[10] Ciafrè SA, Galardi S, Mangiola a, Ferracin M, Liu C-G, Sabatino G, et al. Extensive modulation of a set of microRNAs in primary glioblastoma. Biochemical and biophysical research communications. 2005 Sep 9;334(4):1351-8.

[11] Godlewski J, Nowicki MO, Bronisz A, Williams S, Otsuki A, Nuovo G, et al. Targeting of the Bmi-1 oncogene/stem cell renewal factor by microRNA-128 inhibits glioma proliferation and self-renewal. Cancer research. 2008 Nov 15;68(22):9125-30.

[12] Zhang Y, Chao T, Li R, Liu W, Chen Y, Yan X, et al. MicroRNA-128 inhibits glioma cells proliferation by targeting transcription factor E2F3a. Journal of molecular medicine (Berlin, Germany). 2009 Jan;87(1):43-51.

[13] Shi L, Cheng Z, Zhang J, Li R, Zhao P, Fu Z, et al. hsa-mir-181a and hsa-mir-181b function as tumor suppressors in human glioma cells. Brain research. 2008 Oct 21;1236:185-93.

[14] Wang X-F, Shi Z-M, Wang X-R, Cao L, Wang Y-Y, Zhang J-X, et al. MiR-181d acts as a tumor suppressor in glioma by targeting K-ras and Bcl-2. Journal of cancer research and clinical oncology. 2012 Apr;138(4):573-84.

[15] Cancer T, Atlas G, Information S. Comprehensive genomic characterization defines human glioblastoma genes and core pathways. Nature. 2008 Oct 23;455(7216):1061-8.

[16] Zhang Y, Dutta A. The role of microRNAs in glioma initiation and progression. Frontiers in Bioscience. 2012;700-12.

[17] Pang JC, Kwok WK, Chen Z, Ng H-K. Oncogenic role of microRNAs in brain tumors. Actaneuropathologica. 2009 Jul;117(6):599-611. 
[18] Silber J, James CD, Hodgson JG. microRNAs in gliomas: small regulators of a big problem. Neuromolecular medicine. 2009 Jan;11(3):208-22.

[19] Suh S-S, Yoo JY, Nuovo GJ, Jeon Y-J, Kim S, Lee TJ, et al. MicroRNAs/TP53 feedback circuitry in glioblastomamultiforme. Proceedings of the National Academy of Sciences of the United States of America. 2012 Apr 3;109(14):5316-21.

[20] Duan R, Pak C, Jin P. Single nucleotide polymorphism associated with mature miR-125a alters the processing of pri-miRNA. Human molecular genetics. 2007 May 1;16(9):1124-31.

[21] Xia H, He T, Liu C, Cui Y, Song P, Jin X, et al. Cellular Physiology Biochemistry and Biochemistr y MiR-125b Expression Affects the Proliferation and Apoptosis of Human Glioma Cells by Targeting Bmf. Cellular Physiology and Biochemistry. 2009;100081:347-58.

[22] Shi L, Zhang J, Pan T, Zhou J, Gong W, Liu N, et al. MiR-125b is critical for the suppression of human U251 glioma stem cell proliferation. Brain research. Elsevier B.V.; 2010 Feb 2;1312:120-6.

[23] Cortez MA, Nicoloso MS, Shimizu M, Rossi S, Gopisetty G, Molina JR, et al. miR-29b and miR-125a regulate podoplanin and suppress invasion in glioblastoma. Genes, chromosomes \& cancer. 2010 Nov;49(11):981-90.

[24] Dou T, Wu Q, Chen X, Ribas J, Ni X, Tang C, et al. A polymorphism of microRNA196a genome region was associated with decreased risk of glioma in Chinese population. Journal of cancer research and clinical oncology. 2010 Dec;136(12):18539.

[25] Permuth-Wey J, Thompson RC, Burton Nabors L, Olson JJ, Browning JE, Madden $\mathrm{MH}$, et al. A functional polymorphism in the pre-miR-146a gene is associated with risk and prognosis in adult glioma. Journal of neuro-oncology. 2011 Dec;105(3):63946.

[26] Shu M, Zheng X, Wu S, Lu H, Leng T, Zhu W, et al. Targeting oncogenic miR-335 inhibits growth and invasion of malignant astrocytoma cells. Molecular cancer. BioMed Central Ltd; 2011 Jan;10(1):59.

[27] Nagel R, le Sage C, Diosdado B, van der Waal M, Oude Vrielink J a F, Bolijn A, et al. Regulation of the adenomatous polyposis coli gene by the miR-135 family in colorectal cancer. Cancer research. 2008 Jul 15;68(14):5795-802.

[28] Holleman a, Chung I, Olsen RR, Kwak B, Mizokami a, Saijo N, et al. miR-135a contributes to paclitaxel resistance in tumor cells both in vitro and in vivo. Oncogene. Nature Publishing Group; 2011 May 9;(September 2010):1-13.

[29] Navarro A, Diaz T, Martinez A, Gaya A, Pons A, Gel B, et al. Regulation of JAK2 by miR-135a: prognostic impact in classic Hodgkin lymphoma. Blood. 2009 Oct 1;114(14):2945-51. 
[30] Wu S, Lin Y, Xu D, Chen J, Shu M, Zhou Y, et al. MiR-135a functions as a selective killer of malignant glioma. Oncogene. 2011 Dec 5;31(34):3866-74.

[31] Tavazoie SF, Alarcón C, Oskarsson T, Padua D, Wang Q, Bos PD, et al. Endogenous human microRNAs that suppress breast cancer metastasis. Nature. 2008 Jan 10;451(7175):147-52.

[32] Li Y, Yin W, Wang X, Zhu W, Huang Y, Yan G. Cholera toxin induces malignant glioma cell differentiation via the PKA/CREB pathway. Proceedings of the National Academy of Sciences of the United States of America. 2007 Aug 14;104(33):13438-43.

[33] Shu M, Zhou Y, Zhu W, Wu S, Zheng X, Yan G. Activation of a pro-survival pathway IL-6/JAK2/STAT3 contributes to glial fibrillary acidic protein induction during the cholera toxin-induced differentiation of C6 malignant glioma cells. Molecular oncology. Elsevier B.V; 2011 Jun;5(3):265-72.

[34] Shu M, Zhou Y, Zhu W, Zhang H, Wu S, Chen J, et al. MicroRNA 335 is required for differentiation of malignant glioma cells induced by activation of cAMP/protein kinase A pathway. Molecular pharmacology. 2012 Mar;81(3):292-8.

[35] Baraniskin A, Kuhnhenn J, Schlegel U, Maghnouj A, Zöllner H, Schmiegel W, et al. Identification of microRNAs in the cerebrospinal fluid as biomarker for the diagnosis of glioma. Neuro-oncology. 2012 Jan;14(1):29-33.

[36] Jiang L, Mao P, Song L, Wu J, Huang J, Lin C, et al. miR-182 as a prognostic marker for glioma progression and patient survival. The American journal of pathology. 2010 Jul;177(1):29-38.

[37] Srinivasan S, Patric IRP, Somasundaram K. A ten-microRNA expression signature predicts survival in glioblastoma. PloS one. 2011 Jan;6(3):e17438.

[38] Everhard S, Kaloshi G, Crinière E, Benouaich-Amiel A, Lejeune J, Marie Y, et al. MGMT methylation: a marker of response to temozolomide in low-grade gliomas. Annals of neurology. 2006 Dec;60(6):740-3.

[39] Hegi ME, Diserens A-C, Gorlia T, Hamou M-F, de Tribolet N, Weller M, et al. MGMT gene silencing and benefit from temozolomide in glioblastoma. The New England journal of medicine. 2005 Mar 10;352(10):997-1003.

[40] Stupp R, Hegi ME, Mason WP, van den Bent MJ, Taphoorn MJB, Janzer RC, et al. Effects of radiotherapy with concomitant and adjuvant temozolomide versus radiotherapy alone on survival in glioblastoma in a randomised phase III study: 5-year analysis of the EORTC-NCIC trial. The lancet oncology. 2009 May;10(5):459-66.

[41] Ujifuku K, Mitsutake N, Takakura S, Matsuse M, Saenko V, Suzuki K, et al. miR-195, miR-455-3p and miR-10a( $\left.{ }^{*}\right)$ are implicated in acquired temozolomide resistance in glioblastomamultiforme cells. Cancer letters. 2010 Oct 28;296(2):241-8. 
[42] Slaby O, Lakomy R, Fadrus P, Hrstka R, Kren L, Lzicarova E, et al. MicroRNA-181 family predicts response to concomitant chemoradiotherapy with temozolomide in glioblastoma patients. Neoplasma. 2010 Jan;57(3):264-9.

[43] Li Y, Li W, Yang Y, Lu Y, He C, Hu G, et al. MicroRNA-21 targets LRRFIP1 and contributes to VM-26 resistance in glioblastomamultiforme. Brain research. 2009 Aug 25;1286:13-8.

[44] Ren Y, Zhou X, Mei M, Yuan X-B, Han L, Wang G-X, et al. MicroRNA-21 inhibitor sensitizes human glioblastoma cells U251 (PTEN-mutant) and LN229 (PTEN-wild type) to taxol. BMC cancer. 2010 Jan;10:27.

[45] McElhinney RS, McMurry TBH, Margison GP. O6-alkylguanine-DNA alkyltransferase inactivation in cancer chemotherapy. Mini reviews in medicinal chemistry. 2003 Aug;3(5):471-85.

[46] Sullivan JM. Estrogen replacement therapy. The American Journal of Medicine. 1996 Oct;101(4):56S-60S.

[47] Ferrara N, Hillan KJ, Novotny W. Bevacizumab (Avastin), a humanized anti-VEGF monoclonal antibody for cancer therapy. Biochemical and biophysical research communications. 2005 Jul 29;333(2):328-35.

[48] Costanzo R, Piccirillo MC, Sandomenico C, Carillio G, Montanino A, Daniele G, et al. Gefitinib in non small cell lung cancer. Journal of biomedicine \& biotechnology. 2011 Jan;2011:815269.

[49] Capdeville R, Buchdunger E, Zimmermann J, Matter A. Glivec (STI571, imatinib), a rationally developed, targeted anticancer drug. Nature reviews. Drug discovery. 2002 Jul;1(7):493-502.

[50] Rao SAM, Santosh V, Somasundaram K. Genome-wide expression profiling identifies deregulated miRNAs in malignant astrocytoma. Modern pathologyø: an official journal of the United States and Canadian Academy of Pathology, Inc. 2010 Oct; 23(10):1404-17.

[51] Parsons DW, Jones S, Zhang X, Lin JC-H, Leary RJ, Angenendt P, et al. An integrated genomic analysis of human glioblastomamultiforme. Science (New York, N.Y.). 2008 Sep 26;321(5897):1807-12.

[52] Elmén J, Thonberg H, Ljungberg K, Frieden M, Westergaard M, Xu Y, et al. Locked nucleic acid (LNA) mediated improvements in siRNA stability and functionality. Nucleic acids research. 2005 Jan;33(1):439-47.

[53] Soutschek J, Akinc A, Bramlage B, Charisse K, Constien R, Donoghue M, et al. Therapeutic silencing of an endogenous gene by systemic administration of modified siRNAs. Nature. 2004 Nov 11;432(7014):173-8.

[54] Corey DR. Chemical modification: the key to clinical application of RNA interference? The Journal of clinical investigation. 2007 Dec;117(12):3615-22. 
[55] Whitehead K a, Langer R, Anderson DG. Knocking down barriers: advances in siRNA delivery. Nature reviews. Drug discovery. 2009 Feb;8(2):129-38.

[56] Lee JB, Hong J, Bonner DK, Poon Z, Hammond PT. Self-assembled RNA interference microsponges for efficient siRNA delivery. Nature materials. 2012 Apr;11(4):316-22.

[57] Tao Y, Ruan J, Yeh S-H, Lu X, Wang Y, Zhai W, et al. Rapid growth of a hepatocellular carcinoma and the driving mutations revealed by cell-population genetic analysis of whole-genome data. Proceedings of the National Academy of Sciences of the United States of America. 2011 Jul 19;108(29):12042-7.

[58] Puente XS, Pinyol M, Quesada V, Conde L, Ordóñez GR, Villamor N, et al. Wholegenome sequencing identifies recurrent mutations in chronic lymphocytic leukaemia. Nature. 2011 Jul 7;475(7354):101-5.

[59] Stephens PJ, Tarpey PS, Davies H, Van Loo P, Greenman C, Wedge DC, et al. The landscape of cancer genes and mutational processes in breast cancer. Nature. 2012 Jun 21;486(7403):400-4. 